\title{
Un dialogo non interrotto: Arfé e Salvemini tra storia e politica
}

\author{
Giovanni Scirocco
}

\begin{abstract}
An Uninterrupted Dialogue: Arfé and Salvemini between History and Politics reconstructs the origins of the republication of Salvemini's writings on the Southern question. For the young Neapolitan historian Gaetano Arfé, Salvemini represented a model both as a historian and for his political commitment, above all because of their common interests that underlay their collaboration, albeit only for a brief period: the Southern question, the socialist movement and antifascism.
\end{abstract}

Key words: Arfé, Salvemini, History, Politics, Socialism, Southern question. Parole chiave: Arfé, Salvemini, storia, politica, socialismo, Questione meridionale.

Gaetano Arfé, lo storico napoletano scomparso il 13 settembre 2007, ha avuto il merito di essere, oltre che studioso e interprete, anche testimone e protagonista del tempo che ha vissuto. Autore di opere come i due volumi della Storia dell'Avanti! (Edizioni Avanti!, Milano 1956-58) e della Storia del socialismo italiano 1892-1926 (Einaudi, Torino 1965), fu chiamato a diversi e importanti incarichi politici. Nel 1959 fu nominato condirettore della rivista «Mondo Operaio», carica che conserverà fino al 1971, ricoprendo contemporaneamente, dal 1966 al 1976, la direzione dell' «Avanti!». Proprio a causa delle inchieste sulle "trame nere" pubblicate sul giornale da lui diretto, Arfé fu vittima di un attentato al tritolo che il 2 aprile 1975 devastò la sua abitazione. Nel 1972 era stato nel frattempo eletto senatore per il collegio di Parma e divenne vicepresidente della Commissione istruzione e successivamente della Commissione esteri. Deputato nel 1976 del collegio di ParmaModena-Reggio-Piacenza, entrò a far parte della Commissione affari costituzionali e rappresentò il gruppo socialista nella trattativa sul Concordato. $\mathrm{Nel}$ 1979 fu eletto deputato al Parlamento europeo, dove fu relatore sul tema della politica televisiva europea e promotore della Carta dei diritti delle minoranze etniche e linguistiche. Nel 1985 lasciò il Psi, in disaccordo con la politica craxiana, e nel 1987 fu eletto per l'ultima volta senatore nel collegio di Rimini per la Sinistra indipendente. 
Appartenne quindi a una categoria che ha accomunato personaggi come Garosci, Valiani, Venturi: partigiani, militanti politici e contemporaneamente storici, godendo così di quell'ottica di visuale «leggermente angolata rispetto all'universo» ben descritta da Eric Hobsbawm nella sua autobiografia. In questo senso va visto il rapporto, in Arfé vivissimo, tra il giornalista, il politico e lo studioso, nella lunga fedeltà alla storiografia etico-politica e al senso della responsabilità connesso al mestiere di storico. Una concezione della storia (e della politica) affermata peraltro dallo stesso Arfé:

La spinta a occuparmi di storia mi venne non dall'accademia ma dagli eventi dei quali ero partecipe e dal bisogno che avvertivo di intenderne le genesi e le ragioni e di qui il dovere che sentivo di lavorare e scrivere non soltanto per le corporazioni degli storici, ma anche, e principalmente, per le persone tra le quali vivevo e operavo ${ }^{1}$.

In quest'ambito si collocano i problemi affrontati da uno storico dichiaratamente non neutrale (e critico di quello che, a buon diritto, definì «nichilismo storiografico»): il socialismo come "regola di vita" e l'unità europea come obiettivo (scoperti contemporaneamente in quell'esperienza di «Internazionale della montagna» che fu la Resistenza combattuta in Valtellina ${ }^{2}$; la difesa delle ragioni della Resistenza (per la quale criticò l'utilizzo della categoria di «guerra civile») e della Costituzione (non come esercitazione retorica, ma come problema storico) e la critica serrata nei confronti del revisionismo storiografico e dei suoi caratteri di «offensiva ideologica non più contro un'interpretazione della Resistenza indubbiamente segnata dagli anni, ma contro la Resistenza in sé, per la demolizione del suo ethos politico»${ }^{3}$.

\section{I maestri}

Arfé ha spesso ricordato quelli che riconosceva come suoi maestri di storia, di politica e di vita: primi tra tutti, per un napoletano cresciuto in una famiglia di tradizioni socialiste e antifasciste (il padre, Raffaele, era stato segretario della sezione napoletana del Psi in età giolittiana ${ }^{4}$ e nel 1940 fu arresta-

1 G. Arfé, Storie di storici, «Quaderni del Dipartimento di Scienze storiche e sociali dell'Università degli studi di Salerno», 2004, n. 1, ora in Id., Scritti di storia e politica, a cura di G. Aragno, La Città del Sole, Napoli 2005, p. 61.

2 Cfr. Id., L'Internazionale della montagna, «il manifesto», suppl. del 25 aprile 1994, ora in Id., Scritti di storia e politica cit., pp. 197-205; Id., Sessant'anni dopo, «Il Ponte», maggio 2005, pp. 106-17.

3 Id., L'ultima primavera nella storia d'Europa, «Patria indipendente», 11 ottobre 1992, ora in Id., Scritti di storia e politica cit., p. 171; cfr. anche Lettera aperta a Indro Montanelli, «Il Ponte», luglio 2000, pp. 38-43.

${ }^{4}$ Basandosi sui verbali delle riunioni custoditi dal padre, Arfé scrisse uno dei suoi primi saggi, Per la storia del socialismo napoletano: atti della sezione del Psi dal 1908 al 1911, «Movimento operaio», 1953, n. 2, pp. 201-93. 
to per qualche mese), Filippo Turati ${ }^{5}$ e Benedetto Croce, conosciuto personalmente, nel 1942, a 17 anni, grazie a un libraio amico del padre, Ettore Ceccoli. Croce lo ricevette a Palazzo Filomarino, sottoponendolo ad un «benevolo esame» sulle sue letture e i suoi progetti di studio, suggerendogli infine di leggere De Sanctis, Silvio Spaventa, Omodeo. La visita si concluse con il regalo di

una copia del suo volume sul materialismo storico forse a preventivo antidoto per una prevedibile affezione marxista. A Croce, da sinistra, è stato mosso il rimprovero di avere incitato i giovani, in quegli anni, allo studio e non alla lotta. Non entro nel merito della questione, offro una testimonianza: quando un anno dopo quell'incontro mi trovai, da solo, lontano da casa e separato dal fronte, a decidere il che fare, entrai nelle formazioni partigiane che si intitolavano al motto carducciano di «Giustizia e Libertà» - e il mio Carducci era quello che avevo conosciuto attraverso l'interpretazione di Croce - senza travaglio, con la serenità che mi veniva dalla convinzione che quella decisione avrebbero approvata i due uomini al cui giudizio tenevo di più, mio padre, vecchio socialista, e "don Benedetto", il filosofo della libertà. È un debito di gratitudine che non ho mai dimenticato ${ }^{6}$.

L'analisi del pensiero di Croce fu poi approfondita, dopo la guerra e gli studi universitari, durante gli anni di frequenza dell'Istituto di studi storici napoletano. Nei due anni di corso, dal 1948 al 1950, furono suoi compagni, tra gli altri, Franco Bolgiani, Charles Delzell, Emilio Gabba, Giuseppe Giarrizzo, Nicola Matteucci, Renato Solmi, Giovanni Pirelli, Giuliano Procacci, Maria Adelaide Salvaco, Pasquale Villani, ma, soprattutto, ebbe come guida Federico Chabod:

A Chabod devo quel tanto che ho imparato di regole del mestiere. I suoi corsi di storia della storiografia erano lezioni di storia della cultura e di metodologia storica. Le indicazioni che ci dava quando gli riferivamo delle nostre ricerche erano sempre cariche di suggestioni, schiudevano orizzonti impensati ${ }^{7}$.

Alla Storia della politica estera italiana dal 1870 al 1896 Arfé dedicò una delle sue prime recensioni, sulla rivista di Gianni Bosio ${ }^{8}$ «Movimento ope-

${ }_{5}^{5}$ Cfr. ad es. G. Arfé, Filippo Turati, in M. Degl'Innocenti (a cura di), Filippo Turati e il socialismo europeo, Guida, Napoli 1985, pp. 23-38.

${ }^{6}$ Cfr. E. Romeo (a cura di), La scuola di Croce. Testimonianze sull'Istituto italiano per gli studi storici, il Mulino, Bologna 1992, pp. 137-38.

${ }^{7}$ Ivi, p. 148. Cfr. anche G. Arfé, Per Chabod il mestiere di storico coincise con il mestiere di vivere, «Avanti!», 28 marzo 1961.

8 A Bosio Arfé fu sempre particolarmente affezionato, come dimostra anche la lettera che scrisse a Nenni il 24 agosto 1971, annunciandogliene la morte e ricordandogli come Bosio gli fosse rimasto legato: la lettera è in Archivio centrale dello Stato (ACS), Carte Nenni, b. 17. Cfr. anche la risposta, disillusa e per certi versi profetica, di Nenni: «Per certi uomini che non avevano umiliato la politica al rango del politicantismo, capisco il tormento di chi come te si muove sulla medesima linea. D'altro canto che fare? Non si costruisce qualche cosa se non coi 
raio», in cui, pur lodandone la novità di impostazione metodologica, ne coglieva i limiti nel collocarsi completamente all'interno della storiografia etico-politica, «pur giovandosi di tecnica forse più sottile e di più moderna sensibilità». Così la nascita e lo sviluppo del movimento operaio non acquistava il dovuto rilievo, anche nel quadro d'insieme:

Osservazioni siffatte traggono origine da insoddisfazioni ed esigenze che son vive e reali nella cultura del nostro tempo, e che vanno espresse quando ci si trova di fronte ad un'opera che è indubbiamente un avvenimento nella storia della nostra storiografia. Ed è per questo che la storiografia italiana, nella sua parte più giovane e più avanzata, accoglie con ammirazione e gratitudine, anche se in forma polemica, l'insegnamento dei suoi maestri, eppur si dispone a tentar nuove vie, alla luce di nuovi problemi e di nuove esperienze di vita politica e morale ${ }^{9}$.

Appare evidente la lezione di un altro maestro, Antonio Gramsci, a cui Arfé riconobbe sempre un debito non inferiore a quello di Croce nella formazione del suo modo di vedere e concepire la storia, creazione di uomini «che operano collettivamente e consapevolmente nella realtà, alla luce di una loro cultura, in nome della loro idealità» 10 .

Una lezione che diventò anche impegno politico, nel "gruppo di studio" intitolato allo stesso Gramsci, condotto da Guido Piegari e Gerardo Marotta, sotto l'egida di Nino Cortese. In questo ambito, Arfé tenne due relazioni, la prima nel 1951 dal titolo "La vita italiana nel primo ventennio del Novecento", la seconda, l'anno seguente, dal titolo "Il Mezzogiorno d'Italia dalla repubblica napoletana al 1914". Un'avventura, quella del «Gruppo Gramsci», che si chiuse però rapidamente, per i contrasti sorti tra i giovani comunisti che vi erano impegnati e Giorgio Amendola, che li riteneva legati a Pietro Secchia ${ }^{11}$.

materiali disponibili. E sul valore morale di questi materiali umani stiamo assistendo a crolli paurosi. Non si può reagire che con l'ottimismo della volontà che purtroppo in me sta declinando rendendo amaro l'ultimo periodo della vita» (ibidem). Si comprende quindi perché, in un'altra occasione, Arfé (cui, nella complicata vicenda successiva al licenziamento di Bosio da «Movimento operaio», fu offerta anche la vice-direzione del periodico: cfr. la lettera a Bosio dell'8 gennaio 1954, in Istituto mantovano di storia contemporanea - d'ora in poi IMSC -, Carte Bosio, b. 85) abbia paragonato la figura di Bosio a quella di un altro personaggio a cui fu legato e che incontreremo più avanti, Don Milani, accomunati dalla forma mentis del rivoluzionario, «di chi non è condizionato dalla società in cui vive e vuole realmente cambiarla» (G. Arfé-A. Ricciardi, Dialogo sul socialismo tra militanza politica e ricerca storica, «Il Ponte», novembre 2004, p. 97).

9 «Movimento operaio», settembre-ottobre 1952, pp. 861-64.

${ }^{10}$ E. Romeo (a cura di), La scuola di Croce cit., pp. 150-51.

${ }_{11}$ Cfr. G. Arfé, Storie di storici cit., p. 74. Sulle attività del «Gruppo Gramsci» oltre a E. Rea, Mistero napoletano: vita e morte di un comunista negli anni della guerra fredda, Einaudi, Torino 1995, cfr. il ricordo di G. Marotta, Mi mise tra le mani $i$ "Princìpi di etica" di Bertrando Spaventa, in C. Raia (a cura di), Per Gaetano Arfé. Testimonianze, Libreria Dante\&Descartes, Napoli 2008, pp. 142-45. 


\section{Tra Calamandrei e don Milani}

Nel frattempo, il $1^{\circ}$ luglio 1950, Arfé era entrato in servizio come funzionario degli Archivi di Stato (vincitore di concorso insieme, tra gli altri, a Fausto Fonzi, Elio Lodolini e Gianfranco Merli). La sua prima sede di lavoro fu Genova ${ }^{12}$. Tornato pochi mesi dopo a Napoli, era stato destinato, secondo la relazione dell'8 marzo 1952 del locale sovrintendente, alla Sezione Diplomatica e di Casa Reale, dove si era dedicato allo schedario-indice delle carte della segreteria antica di Casa Reale, all'ordinamento-inventario degli archivi Maresca di Serracapriola e Revertera della Salandra, allo spoglio, trascrizione e collazione degli archivi angioini.

Alla fine del 1952 fu trasferito d'ufficio a Firenze, per la sua attività politica, dal ministero dell'Interno, allora retto da Scelba ${ }^{13}$. Su invito di Giorgio Amendola aveva infatti partecipato a una manifestazione per la pace della "gioventù meridionale", apertasi con i discorsi di Enrico Berlinguer e di Mario Alicata e interrotta per consentire la partecipazione ai funerali di Croce, che si celebravano nello stesso giorno:

L'esibizione mi valse un trasferimento per via telegrafica, firmato da Mario Scelba, da Napoli a Firenze ${ }^{14}$. Il provvedimento mi creava grossi problemi personali e quando gliene parlai Giorgio mi offrì un lavoro di funzionario presso il "Movimento di Rinascita" e mi dette al tempo stesso, con una duplice motivazione, un consiglio: quello di non accettare. La nostra battaglia, mi disse sarà lunga e dura e abbiamo bisogno di compagni che studiino più che di attivisti; e poi, finita l'era del "rivoluzionario professionale", il rapporto di dipendenza da un partito diventa limite all'autonomia del militante, senza contare, aggiunse, che potresti avere a che fare con personaggi come me e peggiori di me senza le garanzie che l'amministrazione dello Stato comunque ti dà. Ne convenni e egli mi munì di quattro lettere, di quelle che un tempo si chiamavano commendatizie, indirizzate a Mario Fabiani, capo dei comunisti fiorentini, a Romano Bilenchi, direttore del «Nuovo Corriere» di Firenze, a Delio Cantimori e a Cesare Luporini, nelle quali mi presentava come un perseguitato politi-

12 Cfr. il rapporto informativo del 10 marzo 1952 del direttore dell' Archivio di Stato di Genova, in ACS, Ministero dell'Interno, Direzione generale degli Archivi di Stato, Divisione del personale, b. 38 .

13 «La cosa mi causò notevoli fastidi e grandi disagi ma, vent'anni dopo, divenuto suo "vice" di minoranza alla presidenza della Commissione esteri del Senato, scherzosamente lo ringraziai» (G. Arfé-A. Ricciardi, Dialogo sul socialismo cit., p. 96).

${ }^{14} \mathrm{Il}$ ministero dell'Interno aveva già da tempo posto sotto osservazione l'attività politica di Arfé. Il $1^{\circ}$ dicembre 1952 il sovrintendente dell'Archivio di Napoli così scriveva all'Ufficio centrale degli Archivi di Stato: «Da quando il detto funzionario apparve tra i candidati del PSI nelle passate elezioni amministrative, ne ho preso continuamente conto, e mi è costantemente risultato che egli non ha mai cercato di diffondere in ufficio idee estremiste. La sua condotta è d'altra parte irreprensibile; di lui sono contenti superiori e colleghi, mentre nei doveri d'ufficio egli è molto diligente. Infine, dai propositi che egli mi ha espresso, di volersi appartare da ogni contesa politica, e per la bontà del suo carattere, ho motivo di augurarmi che egli, come si dimostra buon funzionario, si dimostrerà anche buon cittadino» (ACS, Ministero dell'Interno, Direzione generale degli Archivi di Stato, Divisione del personale, cit., b. 38). 
co meritevole della migliore solidarietà e quelle lettere mi furono preziose ai fini del mio inserimento nella vita della città ${ }^{15}$.

A Firenze lavorò, sotto la guida del sovrintendente Antonio Panella, insigne paleografo, nel gruppo di archivisti incaricato di redigere la Guida degli archivi di stato italiani e l'inventario delle carte del CLN toscano, vero e proprio «apprendistato nella ricerca» ${ }^{16}$. Si inserì subito nella vivace temperie culturale degli anni '50, a contatto con le redazioni di riviste come «Belfagor» di Russo e «Il Ponte» di Calamandrei, Codignola ed Enriques Agnoletti, con cui iniziò una collaborazione durata sino alla morte ${ }^{17}$, nel ricordo, sempre vivo, dei Rosselli ${ }^{18}$. All'influenza di quegli azionisti delusi dalla scelta frontista di Nenni Arfé attribuiva, tra l'altro, la sua adesione, a Palazzo Barberini, al Psli, durata però solo un anno: decisiva era stata infatti la lettura di Buio a mezzogiorno di Koestler, svolta dopo un discorso in cui Codignola aveva denunciato la volontà egemonica del Pci, rinviando a un futuro indefinito il compimento del suo processo di democratizzazione ${ }^{19}$. Il legame con il gruppo del «Ponte» fu quindi culturale e politico nello stesso tempo. L'accettazione da parte di Saragat di quella che passerà alla storia come la "legge truffa" provocò la rottura dei rapporti con il leader socialdemocratico di Calamandrei e Codignola, che diedero vita al movimento di «Unità popolare», decisivo per impedire, nelle elezioni del 1953, lo scatto della legge ${ }^{20}$.

15 G. Arfé, 35 anni di ricordi con Giorgio Amendola, «L'Antifascista», agosto-settembre 2000. Cfr. anche l'intervista ad A. Zanuttini in L'Archivio centrale dello Stato, 1953-1993, Ministero per i Beni culturali e ambientali, Ufficio centrale per i beni archivistici, Roma 1993, pp. 456-63.

16 G. Arfé-A. Ricciardi, Dialogo sul socialismo cit., p. 95 . Nel 1957 fu distaccato dall'Archivio di Stato e affiancato a Carlo Francovich, direttore dell'Istituto storico della Resistenza in Toscana, con l'incarico di procedere alla sistemazione del materiale archivistico dell' Istituto (cfr. ISRT, Verbali del Comitato direttivo, 8 e 14 giugno 1957 e M.G. Rossi, Luci e ombre di una storia, in L'Istituto Storico della Resistenza in Toscana. Mezzo secolo di vita e di attività, Polistampa, Firenze 2006, p. 50).

17 Il suo primo intervento risale al fascicolo del maggio 1954, con una recensione a R. Balducci, Alessandro Balducci e gli albori del socialismo nel Forlivese (1880-1904), Garzanti, Milano 1954. Nel marzo 1955 recensirà L. Preti, Le lotte agrarie nella valle padana, Einaudi, Torino 1955. Nello stesso anno, rispettivamente nei numeri di giugno e luglio, recensirà A. Borghi, Mezzo secolo di anarchia (1898-1945), con prefazione di G. Salvemini, Esi, Napoli 1954, e il volume collettaneo, edito da Laterza, Dieci anni dopo. 1945-1955. Infine, nel dicembre (pp. 2001-13), pubblicherà il saggio Il meridionalismo di Gaetano Salvemini.

18 «Un altro episodio per me significativo fu quando Nello Traquandi, taciturno e riservato, mi offrì di accompagnarlo al cimitero di Trespiano per "salutare" Nello e Carlo Rosselli, con il quale aveva collaborato prima di condividere la galera con Ernesto Rossi» (G. Arfé-A. Ricciardi, Dialogo sul socialismo cit., p. 97).

${ }^{19}$ Cfr. Problemi di storia del socialismo italiano, intervista a cura di F. Gentile, «Il Ponte», febbraio-marzo 2004. Su Codignola cfr. il ricordo di Arfé, Sulla via della dissidenza, «Il Ponte», novembre 1993

${ }^{20}$ G. Arfé-A. Ricciardi, Dialogo sul socialismo cit., p. 98. In una lettera a Nenni del 25 ottobre 1957 Arfé sostenne, con toni polemici, l'ingresso nel Partito del gruppo di Unità popolare, che poi avverrà alla fine dello stesso anno, arrivando a ventilare le sue dimissioni dal Co- 
Ma le relazioni fiorentine di Arfé si estesero anche agli ambienti cattolici, caratterizzati dalla presenza di Giorgio La Pira, Nicola Pistelli e don Lorenzo Milani (conosciuto grazie a Marcello Del Piazzo, militante dell'Azione Cattolica, che lavorava presso l'Archivio di Stato di Firenze) nella cui scuola, a San Donato, terrà alcune lezioni ${ }^{21}$ e di cui conserverà sempre un ricordo vivissimo (nel 1975 reciterà anche, nel ruolo di se stesso, con Amintore Fanfani e Giorgio La Pira, nel film Don Milani del regista Ivan Angeli):

Una volta parlando della storia del socialismo don Milani mi domandò se era vero che fossero stati i socialisti a provocare la disfatta di Caporetto. Io gli risposi che sulla base delle testimonianze storiche e della storiografia più accreditata non era stato quello l'elemento determinante della sconfitta. È un'illusione che tu ci togli - replicò perché noi, e in particolare questi giovani, avevamo sempre creduto che il partito socialista fosse stato contro la guerra fino a questo punto, fino a provocare una disfatta. Erano cose che in quell'epoca suonavano veramente strane soprattutto dette da un sacerdote $^{22}$.

Furono quindi anni (e incontri) decisivi per la sua formazione di studioso. A Firenze resterà sempre legato: vi tornerà ad insegnare nel 1973, nella Facoltà di Scienze politiche, su richiesta di Giovanni Spadolini e nel 1975 parteciperà alla fondazione dell'Istituto socialista di studi storici 23 .

mitato centrale, «il che può interessarti poco se tu ritieni che le proteste oscure dei "profeti disarmati" valgano poco; può interessarti di più se ti soffermerai a domandarti che cosa realmente rappresentino, quali forze abbiano dietro di sé coloro i quali consumano il loro tempo e le loro energie non esuberanti nei pettegolezzi e negli intrighi degli ambulacri direzionali; può interessarti infine moltissimo se tu pensi alle tue responsabilità del recente passato, delle quali nessuno intende chiederti conto perché esse nacquero da discutibili valutazioni politiche e non da deformazioni ideologiche e morali, ma che finirebbero con lo schiacciarti sul piano storico se tu, dopo avere indicato la grande via della ripresa, dessi l'avallo del tuo nome ad una miserevole politica di setta e di bottega, che chiude la porta in faccia ad una corrente che porta con sé l'eredità di Carlo Rosselli, di Gaetano Salvemini e di Piero Calamandrei, per non ricordare che i morti. Non ti dispiaccia la franchezza di cui ho fatto uso, e considerala un atto di stima delle tue qualità umane e di fiducia politica persistente. "Dixi et servavi animam meam": è un motto anche questo della nostra tradizione» (ACS, Carte Nenni, b. 17).

${ }_{21}$ Cfr. C. Raia, Gaetano Arfé. Un socialista del mio paese, Lacaita, Manduria-Bari-Roma 2003, p. 61.

22 Cfr. «Antologia Vieusseux», aprile-giugno 1982, pp. 10-11 e V. Parlato, Arfé e Milani, fertile incontro, «il manifesto», 13 ottobre 2007. Di don Milani, nel numero dell'ottobre 1958 de «Il Ponte», Arfé recensirà Esperienze pastorali, cogliendo pienamente la particolare posizione del suo autore: «Il problema politico in sé a lui non interessa. Gli manca del tutto il senso liberale e laico dell'autonomia della lotta politica, della immanenza in essa di originali valori morali. I problemi di libertà e di giustizia lo interessano solo in quanto la mancata risoluzione di essi costituisce un ostacolo, in larga parte insormontabile, all'adempimento della sua missione sacerdotale». Don Milani aveva inviato il manoscritto ad Arfé il 20 marzo 1956 e il libro il 5 maggio 1958, con due lettere di accompagnamento che ora si possono leggere in N. Fallaci, Vita del prete Lorenzo Milani. Dalla parte dell'ultimo, Rizzoli, Milano 1993, pp. 555-60.

${ }^{23}$ Cfr. M. Degl'Innocenti, Gaetano Arfé e la Fondazione di studi storici "Filippo Turati", in C. Raia (a cura di), Per Gaetano Arfé. Testimonianze cit., pp. 102-08. 


\section{Salvemini e la questione meridionale}

Un ruolo particolare lo ebbe Salvemini, con cui Arfé entrò in contatto nel 1953 grazie a Franco Venturi, che suggerì a Salvemini di avvalersi della sua collaborazione per la pubblicazione, proposta da Giulio Einaudi ${ }^{24}$, nell'ambito di una ristampa delle opere dello storico di Molfetta, di una nuova edizione del volume Tendenze vecchie e necessità nuove del movimento operaio italiano, pubblicato da Cappelli nel 1922 e ormai introvabile, dedicato «alla polemica, non dottrinale, ma politica contro il riformismo meramente sociale dei sindacati, delle cooperative e delle amministrazioni comunali socialiste del Nord nell'età giolittiana, in nome di un riformismo politico ispirato ai principii e ai valori del socialismo democratico e gradualista» 25 :

Carissimo Salvemini, appena ritornato da Cagliari ho cercato di informarmi per trovare un giovane capace di collaborare con te, l'anno prossimo, nella ricerca dei tuoi articoli sul movimento socialista. Ne ho scritto anche a Leo Valiani, mi sono informato presso altri ed in conclusione vorrei proporti la persona di Gaetano Arfé. È un giovane archivista che è stato recentemente trasferito (tra l'altro per ragioni politiche) da Napoli a Firenze. Ha collaborato a «Movimento Operaio» con saggi che mi paiono interessanti. Il suo interesse è concentrato sulla storia del socialismo ed in genere sull'Italia contemporanea. Il suo stesso mestiere, che credo egli faccia molto bene, dimostra la sua preparazione archivistico-storica. Ha una moglie, intelligente, che è professoressa nelle scuole medie, ma che ora è libera perché non ha ancora potuto ottenere il trasferimento a Firenze. Tutti e due sono persone simpatiche, fini, e che naturalmente sarebbero felicissimi di poter collaborare con te in questo lavoro ${ }^{26}$.

\footnotetext{
24 «Nell'ultima riunione dei collaboratori di casa Einaudi, parlando dei futuri libri di storia da pubblicare, Giulio Einaudi stesso mi ha chiesto se pensavo che tu saresti disposto a veder pubblicata nella collezione storica (quella dove recentemente è uscito il libro di Braudel e dove verrà pubblicata l'opera postuma di Nello Rosselli) una raccolta delle tue opere di carattere storico. Naturalmente la scelta e l'ampiezza della raccolta sono a tuo completo giudizio. Se tu non hai nulla in contrario, Giulio Einaudi preferirebbe forse iniziare la serie dagli scritti sul medioevo, anche per dare una maggiore organicità alla pubblicazione stessa. Naturalmente ho risposto che non sapevo le tue intenzioni in proposito, ma che ti avrei subito scritto per chiedertele. Inutile che ti dica con quanto interesse tutti noi vedremmo riuniti e raccolti da te, sotto la tua cura, i tuoi scritti storici» (F. Venturi a G. Salvemini, 6 ottobre 1953, in ISRT, Carte Salvemini, b. 109; sull' "operazione Salvemini", cfr. anche L. Mangoni, Pensare i libri. La casa editrice Einaudi dagli anni Trenta agli anni Sessanta, Bollati Boringhieri, Torino 1999, pp. 787-88).

25 G. Arfé, La legge del non mollare, «Il Ponte», aprile 2001.

26 Venturi a Salvemini, 14 novembre 1953, in ISRT, Carte Salvemini, b. 109, cit. Cfr. anche la lettera di Venturi a Valiani del 4 novembre 1953, in L. Valiani-F. Venturi, Lettere 1943-1979, a cura di E. Tortarolo, La Nuova Italia, Scandicci 1999, p. 129. La stima di Venturi nei confronti di Arfé (a cui proporrà di curare, sempre per Einaudi, la pubblicazione delle opere di Matteotti: cfr. L. Mangoni, Pensare i libri cit., p. 853n) è testimoniata da una lettera a Valiani del 30 agosto 1957, sempre relativa alla pubblicazione delle opere di Salvemini: «Tenete conto anche che Arfé ha fatto ottimamente l'opera meridionalista e che si potrebbe utilizzare per altro. Aveva proposto a Salvemini - noi entusiasti qui - di curar una raccolta di suoi scritti vecchi sui cattolici (e sarebbe indispensabile oggi...). Ma Salvemini rimandò» (L. Valiani-F. Venturi, Lettere 1943-1979 cit., p. 227).
} 
Fu però lo stesso Salvemini a proporre qualcosa di piuttosto diverso ${ }^{27}$, in una lettera del 2 gennaio 1954:

Franco Venturi mi scrisse il 28 ottobre (!) e il 14 novembre (!) che Einaudi pubblicherebbe volentieri una raccolta dei miei scritti introvabili sul movimento socialista italiano, e che tu e la tua signora sareste lieti di aiutarmi nel lavoro di raccolta. Venturi avrebbe voluto che tu venissi a trovarmi a Firenze. Ma non potremo vederci che a maggio: ché io ho paura del freddo come del mio peggior nemico. potremmo però intenderci per lettera. Anzitutto, io mi domando se i miei scritti servirebbero davvero a una storia del socialismo italiano, o non formerebbero piuttosto un volume interessante su La questione meridionale vista da un socialista dal 1895 al 1953. In fondo il socialismo della Cina, dell'India, del pianeta Marte, mi ha sempre interessato poco. Mi ha interessato solamente il socialismo meridionale ed il socialismo italiano in funzione di quello meridionale e viceversa. Una raccolta di miei scritti su quest'argomento, preceduta da una prefazione sullo sviluppo del mio pensiero in questi sessant'anni, credo che riuscirebbe di un certo interesse [...] Naturalmente tu e la tua signora dovreste essere compensati per questo lavoro. A me pare che la cifra più naturale è che voi prendeste tutti i diritti d'autore. Io vi dirigerei nel lavoro per amor della gloria ${ }^{28}$.

È un tema che ovviamente interessava Arfé come storico, come socialista e come meridionale, almeno dagli anni della frequentazione dell'Istituto italiano per gli studi storici e della militanza nel «Movimento per la rinascita del Mezzogiorno» ${ }^{29}$, sopravvissuto allo scioglimento del Fronte popolare:

Gli influssi di quelle discussioni si possono ritrovare anche nel libro di Romeo sul Risorgimento in Sicilia, che rinnova la problematica storiografica relativa al Risorgimento meridionale, alla luce di una moderna metodologia cui non è estranea la lezione di Gramsci. Lo rilevai già allora scrivendo di quel libro sull'«Avanti!» e conservo ancora un suo biglietto di ringraziamento. Il titolo del mio articolo era Il Risorgimento in Sicilia ${ }^{30}$ e altrove e il giudizio sul libro era che esso superava tutti i limiti della storia regionale per diventare contributo a una nuova, moderna interpretazione del Risorgimento. Sul Risorgimento meridionale ebbi ancora con lui lunghe conversazioni. Avevo in mente un ambizioso progetto di studio sulla classe dirigente meridionale, quale si presentava al momento dell'unità, e sulla parte che aveva avuta all'atto

27 «Riletto il libro, Salvemini aveva pensato di arricchirlo con una serie di articoli sulla questione meridionale antichi e recenti, che rendessero evidente il nesso, per certi aspetti ancora politicamente attuale, tra l'antiriformismo, come da lui inteso, l'antigiolittismo e il meridionalismo» (G. Arfé, Prefazione, a G. Salvemini, Movimento socialista e questione meridionale, Feltrinelli, Milano 1963, p. IX, poi in G. Arfé, I socialisti del mio secolo, a cura di D. Cherubini, Lacaita, Manduria-Bari-Roma 2002, p. 105. La Prefazione della Cherubini, in particolare le pp. 22-25, è utile per inserire il rapporto con Salvemini nel quadro del periodo trascorso da Arfé a Firenze).

${ }^{28} \mathrm{La}$ lettera, in Fondazione Turati (d'ora in poi FT), Carte Arfé, Corrispondenza, b. 1, è parzialmente trascritta nella citata Prefazione di Arfé.

${ }_{29}$ Cfr. G. Arfé, La sinistra meridionale nel dopoguerra, «La Città nuova», 1990, n. 1, pp. 51-66.

30 Pubblicato anche su «Movimento operaio», gennaio-febbraio 1952, pp. 699-702. 
del proprio inserimento nella vita dello Stato unitario. Discutemmo ipotesi di lavoro e metodologie di ricerca. Un concorso, vinto, negli archivi, mi portò via da Napoli. Di tutto quel lavorio rimasero un breve saggio sull'hegelismo napoletano e sulla dottrina dello Stato di Bertrando Spaventa ${ }^{31}$, un altro sul giornalismo politico nella Napoli del $1860^{32}$, una lunga serie di schede sulle elezioni politiche meridionali del 1861 e su alcuni episodi del brigantaggio ${ }^{33}$.

Il primo incontro tra i due, nella camera della pensione abitata da Salvemini in via San Gallo, è stato ricordato ancora pochi anni fa da Arfé, in un ritratto vivacissimo, ricco di quel gusto, a lui consueto e per il quale ha sempre riconosciuto di essersi ispirato a Croce, del particolare e dell'aneddoto non fine a se stesso:

Da me volle saper tutto su quanto avevo visto nella guerra partigiana ${ }^{34}$, e in particolare sul rapporto con le popolazioni tra le quali operavamo [...]; sull'Istituto italiano di studi storici, che avevo frequentato per tre anni sotto l'ombra paternalisticamente protettiva di Benedetto Croce ${ }^{35}$; sulle mie letture e sui miei studi e si entusiasmò quando gli dissi che avevo avviata una ricerca, rimasta incompiuta, sulle elezioni del 1861 nel Mezzogiorno. A coronamento degli incontri mi offrì di collaborare con lui alla raccolta dei suoi scritti per la quale, tramite Venturi, si era impegnato con Einaudi. Riteneva opportuno, aggiunse subito, che il mio nome non comparisse quale curatore perché, giovane com'ero, mi sarei tirato precocemente addosso l'avversione di tre quarti del mondo accademico, della setta crociana e dell'intero partito comunista. La rivalutazione del giolittismo alla quale stavamo assistendo e che vedeva convergere, con Croce e col fiore dell'accademia italiana, Giovanni Ansaldo e Palmiro Togliatti, confermava che la sua preoccupazione non era infondata ${ }^{36}$.

${ }^{31}$ G. Arfé, L'hegelismo napoletano e Bertrando Spaventa, «Società», marzo 1952, pp. 3-20.

32 Id., Note sul giornalismo napoletano nella crisi del 1860, «Annali della Facoltà di Lettere e filosofia dell'Università di Napoli», 1951, pp. 1-34.

${ }^{33}$ Cfr. E. Romeo (a cura di), La scuola di Croce cit., pp. 139-40.

34 È un tema che tornerà spesso nei loro scambi epistolari, con notazioni sorprendenti per chi abbia conosciuto lo sguardo mite e buono di Arfé: «Caro Professore, grazie infinite degli auguri e delle buone cose che mi dice. Spero che mia figlia possa vedere un mondo almeno un pochino migliore. Io a diciotto anni mi sono trovato in galera ed a venti ero un esperto in ogni tipo d'arma da fuoco, ma non avevo ancora messo piede in un'aula universitaria. E quando l'ho fatto (università di Napoli!) mi è accaduto più volte di rimpiangere il mitra. Però poi la vita riprende sempre il suo corso e chi ne ha voglia cammina» (ISRT, Carte Salvemini, b. 92, lettera del 5 settembre 1954).

35 «Di Croce Salvemini era fiero avversario [...]. È difficile immaginare due intelligenze e due temperamenti più diversi tra loro. Quando seppe che ero stato allievo dell'Istituto volle che lo informassi dettagliatamente su Chabod, del quale faceva gran conto come storico e al quale era legato da un debito di gratitudine perché era stato Chabod ad aiutarlo quando, liberato dal carcere, era evaso dall'Italia. Mi commiserò - "disgraziato!" - quando seppe che avevo letto e studiato l'opera filosofica di Croce. Si consolò quando lo assicurai che non portavo lo stesso amore a Gentile» (E. Romeo, a cura di, La scuola di Croce cit., pp. 149-50).

36 G. Arfé, Nota a G. Salvemini, Il ministro della mala vita: notizie e documenti sulle elezioni giolittiane nell'Italia meridionale, a cura di S. Bucchi, Bollati Boringhieri, Torino 2000. La frequentazione con Salvemini «fu l'occasione, via via che il lavoro procedeva, a una serie di vivacissime rievocazioni di uomini e fatti dell'Italia giolittiana. La carica di passionalità era 
Salvemini si appassionò alla ricerca dei propri articoli per la raccolta $(« \mathrm{Ca}-$ ro Arfé, questi articoli sono come le ciliegie», gli scrisse il 14 agosto 195437), in primo luogo perché lo riportavano ai tempi della propria giovanile militanza politica:

Ho finito di dare un'occhiata al materiale da te raccolto e già selezionato. Credo che quel lavoro vale (sic) la pena di essere stato fatto, perché può dare elementi allo studio dell'Italia nel quindicennio dal 1897 al 1913 [...]. Mangiando viene l'appetito. Nel 1910 (se la memoria non m'inganna) ebbi una polemica con l'intero gruppo parlamentare socialista. Fu riprodotta in Tendenze vecchie e necessità nuove. Credo sia stato prima del Congresso di Milano. Il mio discorso al Congresso di Milano lo abbiamo debitamente soppresso. Ma una parte di quella polemica va forse riesumata. È un lungo articolo in cui enumeravo le prove della degenerazione oligarchica avvenuta nel gruppo parlamentare socialista italiano. Vedi se ti pare sia il caso di dissotterrare anche questo cadavere. Nella nostra collezione non c'è nulla del 1910. Invece il 1910 fu uno dei più battaglieri. Se non riproduciamo almeno quell'articolo daremo l'impressione che io abbia dato tregua ai deputati socialisti ${ }^{38}$.

Quegli articoli toccavano poi temi che Salvemini sentiva ancora come attuali, anche per la rinnovata importanza assunta, nei primi anni del secondo dopoguerra, dalla questione meridionale, grazie alla presenza, per la prima volta, di un movimento di massa e diffuso sul territorio «nel quale militavano, a fianco del proletariato contadino e operaio, giovani intellettuali refrattari alle tentazioni del trasformismo e tra essi - fatto che lo aveva colpito moltissimo, per cui l'interrogatorio su questo si estese anche a mia moglie - molte ragazze che nel vecchio movimento socialista si contavano sulle punte delle dita» ${ }^{39}$. Le incertezze, i dubbi, le perplessità di Salvemini nel corso del lavoro furono comunque frequenti, e non solo di carattere storiografico:

Continuo certi momenti a domandarmi se valeva la pena di mettere insieme tanti documenti di archeologia politica, quasi che fosse per me il caso di ripetere: exegi monumentum aere perennius. Perciò vorrei che tu e Venturi decidiate caso per caso sugli articoli da sopprimere (magari sopprimerli tutti) e quelli da pubblicare. Io proprio mi sento inetto a decidere, perché oscillo fra il sospetto di soffrire di vanità senile come F.S. Nitti e il masochismo ${ }^{40}$.

Salvemini si mostrò, nel complesso, per quanto lo permettesse un carattere irrequieto e una personalità vulcanica, anche negli anni della vecchiaia, abbastanza "docile" nei confronti dei suggerimenti e dei consigli dei due più giovani storici ( Va bene per gli scritti rifiutati. Tutto ciò che non metto - diceva

tale che sembrava parlasse di vicende accadute il giorno prima. Giolitti veniva gratificato di epiteti non tutti riferibili»: E. Romeo (a cura di), La scuola di Croce cit., p. 150.

${ }^{37}$ FT, Carte Arfé, Corrispondenza, b. 1.

38 Ivi, lettera del 17 agosto 1954.

${ }^{39}$ G. Arfé, La legge del non mollare cit.

40 FT, Carte Arfé, Corrispondenza, b. 1, lettera del 19 settembre 1954. 
Verdi - non corre il rischio di essere fischiato» ${ }^{41}$ ). Il 19 maggio 1955 Arfé poteva quindi finalmente scrivere a Venturi, che aveva costantemente informato sugli sviluppi della pubblicazione:

Come avrà saputo il lavoro è finito e non c'è che da aspettare l'uscita dei volumi. Quest'ultima fase è stata un po' laboriosa per le ultime rifiniture. Ho compilato le note che Salvemini in un primo tempo aveva categoricamente escluse (disturbano il lettore e non giovano allo studioso) e ho contenuto entro limiti ragionevoli le ultime notevoli pretese di cambiamento [...]. A documento di queste e delle precedenti discussioni mi restano sessantotto lettere di Salvemini che saranno la fonte per la storia di questi volumi ${ }^{42}$.

Il volume fu pubblicato da Einaudi nel 1955 con il titolo Scritti sulla questione meridionale (1896-1955) per poi diventare, nel 1963, con il titolo Movimento socialista e questione meridionale, uno dei volumi, curato dallo stesso Arfé, della ristampa Feltrinelli delle opere complete di Salvemini ${ }^{43}$.

La figura di Salvemini legò quindi tra di loro, per il giovane storico napoletano, una serie di interessi storiografici (il socialismo degli inizi del secolo, il meridionalismo, l'antifascismo di «Giustizia e Libertà») rappresentati dai ricordi di persone reali, in carne ed ossa (e parole). Un'esperienza fondamentale anche per i suoi futuri studi di storia del socialismo, per la possibilità di lavorare con un uomo che aveva conosciuto da vicino i capi del socialismo italiano, da Morgari a Modigliani, da Turati a Bissolati ${ }^{44}$.

Si comprende perché Arfé abbia spesso ricordato con piacere «la prorompente carica di umanità, velata dall'ironia» di Salvemini, che difese sempre dalla critica, spesso rivoltagli, di moralismo. Si trattava invece, secondo Arfé, di un forte senso della distinzione tra il vero e il falso, il giusto e l'ingiusto, regola di vita e principale criterio di interpretazione della realtà nei confronti del cinismo e dell' "apotismo" elevati a norma di comportamento e di atteggiamento storiografico ${ }^{45}$. Salvemini rappresentò quindi, agli occhi di Arfé, la figura, oltre che di un maestro di storia, di etica intellettuale, a confronto di una tradizione meno nobile, di voltagabbana o fascisti tout court:

${ }^{41}$ Ivi, lettera del 28 dicembre 1954.

${ }^{42}$ La lettera è in Archivio privato Franco Venturi, Torino. Ringrazio Antonello Venturi per la cortesia con cui ha messo a mia disposizione le lettere di Arfé al padre.

${ }^{43}$ I motivi del passaggio da Einaudi a Feltrinelli sono chiariti in una lettera riservatissima (e, per certi versi, preveggente) di Ernesto Rossi a Giorgio Agosti, datata 23 settembre 1957: «Credo che la situazione finanziaria della società Einaudi sia molto più grave di quello che tu scrivi. Persone bene informate mi dicono che è disastrosa [...] Mio timore è che Mondadori (o un altro editore affarista del suo tipo) rilevi domani la Einaudi per un boccone di pane, mantenga il nome, ma ne cambi l'indirizzo» (E. Rossi, Epistolario 1943-1967. Dal Partito d'Azione al centro-sinistra, a cura di M. Franzinelli, Laterza, Roma-Bari 2007, p. 277; cfr. anche L. Mangoni, Pensare i libri cit., p. 787n).

${ }^{44}$ G. Arfé, Prefazione, in G. Salvemini, Movimento socialista e questione meridionale cit.

${ }^{45} \mathrm{Cfr}$. G. Arfé, La legge del non mollare cit. 
Carissimo Professore, mi permetto una osservazione alla Sua ultima cartolina: ben più di quattro o cinque giovani si sono incontrati ogni anno idealmente con Lei durante il fascismo e ne hanno tratto le conseguenze del caso. In tempi che erano quelli che erano Lei ha smosso quello che poteva smuovere e non è stato poco se si pensi a quanti e soprattutto quali sono stati gli uomini della lotta antifascista formati direttamente o indirettamente da Lei. E tutti questi, ed io tra gli ultimi arrivati mi associo a loro, conservano per Lei un senso vivo di gratitudine e di affetto. In fin dei conti il fascismo, tra i tanti enormi malanni, ci ha portato questo di positivo, ci ha permesso di renderci conto delle qualità vere degli uomini. Senza il fascismo i Volpe e i Gentile, i Prezzolini e gli Ansaldo sarebbero rimaste persone dabbene, fino alla fine dei loro giorni. E invece la tempesta ci ha permesso di vedere gli uomini così come veramente erano, ed è enorme il valore morale che ha avuto per molti di noi l'insegnamento di onestà ad ogni costo, personale e politico, che ci è venuto dai maestri, più che capi dell'antifascismo italiano ${ }^{46}$.

L'atteggiamento di Arfé nei confronti del vecchio maestro fu, all'inizio, oltre che improntato al naturale rispetto, addirittura quasi reverenziale, anche per una certa aura di leggenda che, nonostante i passati contrasti, accompagnava Salvemini all'interno del mondo socialista:

Giorni fa sono stato a Torino ad assistere al congresso socialista, meno grigio dei passati, ed a prender parte ad una riunione promossa da Pertini tra ex-partigiani delle "Matteotti" e delle "Gl" per organizzare una raccolta di materiale documentario relativo alla storia della Resistenza, da porre a disposizione degli studiosi. Pertini la saluta e m'ha raccontato che nel ' 44 fu a Molfetta a parlare in una sezione socialista, dove trovò il Suo ritratto e quando parlò di Lei vi fu chi pianse tra i contadini presenti ${ }^{47}$.

Ma la tempra di storico prese rapidamente il sopravvento e la discussione si spostò sui temi della redazione della raccolta di scritti di Salvemini, il socialismo e la questione meridionale:

A proposito della introduzione mi permetto infine qualche osservazione. Mi è molto piaciuta la prima parte, nella quale rilevo una sola cosa: quando parla del gruppo di socialisti torinesi che nel '14 Le offrì la candidatura a Torino, Lei non ricorda che in quel gruppo c'era Gramsci e il futuro stato maggiore del partito comunista. Mi pare che la cosa sia da ricordare perché è un fatto storicamente rilevante che il gruppo più intelligente di socialisti del tempo simpatizzasse per la Sua campagna; ed è anche importante perché, tramite Gramsci e il suo saggio sulla "Questione meridionale", pur entro una impostazione radicalmente diversa, i risultati della Sua analisi sono entrati a far parte del bagaglio mentale degli attuali dirigenti comunisti meridionali ${ }^{48}$.

\section{E ancora, il 18 maggio 1955:}

Ho spedito a Serini i resti delle bozze e non c'è ora che da attendere l'uscita dei volumi che avverrà sicuramente entro giugno. C'è solo da dire che è un peccato che $\mathrm{i}$

\footnotetext{
46 ISRT, Carte Salvemini, b. 92, lettera del 10 gennaio 1955

47 Ivi, lettera del 9 aprile 1955.

48 Ivi, lettera del 9 gennaio 1955.
} 
volumi non siano usciti dieci anni fa. Ricordo che allora noi giovani che dopo anni sempre più o meno amari venivamo affacciandosi alla vita politica eravamo alla ricerca infruttuosa di un orientamento. Mai forse come allora i giovani meridionali ebbero una spinta alla politica così impetuosa e disinteressata. Di Suo non c'era che la raccolta del Cappelli del '22 (ce n'era a Napoli una sola copia, credo, e notevolmente mutilata) e poi Dorso a grandi dosi, ma era astruso, astratto e incoraggiava un certo gusto agli alchimismi politici che di incoraggiamento non aveva bisogno. Il saggio di Gramsci fu allora una rivelazione e valse ai comunisti la conquista di un'intera leva di giovani di prim'ordine. Io fui tra i pochissimi a rimanere modestamente socialista. Comunisti diventarono molti dei miei compagni e la gran maggioranza dei giovani del partito d'azione ${ }^{49}$.

Non stupisce, dunque, che per Arfé l'adesione di Salvemini al socialismo non soltanto preceda cronologicamente l'impegno meridionalistico, ma ne costituisca la premessa e ne indirizzi il corso: dal marxismo gli vengono i criteri di metodo che egli originalmente applica allo studio della società meridionale; dal messianismo socialista gli viene quella religione laica che egli scoprì a Firenze e di cui a Firenze rievocò la scoperta, quando ritornò sulla cattedra che il fascismo gli aveva strappata ${ }^{50}$.

Eppure, Arfé non abbraccerà mai in pieno la tesi di Lelio Basso sulle due fasi dell'attività di Salvemini ${ }^{51}$, separate nettamente tra loro dall'abbandono del Psi e del marxismo. Anzi, a suo parere, al di là delle divergenze evidenti nelle due fasi, soprattutto sul ruolo dei partiti, l'opera di Salvemini è caratterizzata da una sostanziale continuità di temi e di metodo:

Non muta, soprattutto, l'ispirazione ideale di Salvemini, fatta di quel socialismo scoperto e costruito in anni lontani, in cui Marx si era accoppiato a Cattaneo, a Mazzini, forse anche ai filosofi dell'illuminismo: un socialismo nel quale l'elemento etico, mai sbandierato, prevale nettamente su quello ideologico, un socialismo senza palingenesi e senza quindi la mistica del partito che vi si accompagna [...]. I fili col movimento socialista, in sostanza, non risultano spezzati. Un motivo ideale unitario salda tutte le fasi dell'attività di Salvemini ${ }^{52}$.

\footnotetext{
49 Ibidem. La forte presenza di militanti comunisti nel Movimento per la rinascita del Mezzogiorno era stato oggetto di lunghe discussioni tra Arfé e Salvemini, convinto che fosse necessario parlare a quei giovani «perché prendessero coscienza dell'insanabile contraddizione nella quale si trovavano a operare e si indirizzassero verso un socialismo autenticamente riformista che non ripercorresse la strada, complici, questa volta, per spregiudicate ragioni tattiche i comunisti, segnata a suo tempo da Giolitti» (G. Arfé, La legge del non mollare cit.).

${ }^{50}$ G. Arfé, Prefazione cit., pp. XII-XIII.

${ }^{51}$ L. Basso, Gaetano Salvemini socialista e meridionalista, Lacaita, Manduria 1959 (cfr. la recensione di Arfé in «Critica sociale», 5 marzo 1960, pp. 129-30 e l'interpretazione, per certi versi speculare e opposta a quella di Basso, di G. Quagliariello, Il Salvemini di Lelio Basso, in Id., a cura di, Cultura laica e impegno civile. Quarant'anni di attività di Piero Lacaita Editore, Lacaita, Manduria-Bari-Roma 1990, pp. 97-116).

52 G. Arfé, Prefazione cit., pp. XIII-XIV.
} 


\section{«Il calendario dei morti»: un progetto incompiuto}

Salvemini fu evidentemente molto soddisfatto della collaborazione con Arfé, così da proporre, a lui e a sua moglie, un nuovo lavoro, destinato però a restare incompiuto:

Mettendo in ordine una cassa di vecchie carte, ho trovato tutti gli appunti che presi, o meglio feci prendere una volta, per accertare su parecchi quotidiani quanta gente i fascisti avevano ammazzato dal 1919 al 1922, 28 ottobre. E lavoro enorme, che non vorrei andasse perduto, anche se per mettere insieme la lista dovessi spendere un centinaio di migliaia di lire. Il lavoro deve essere fatto da persona, non solo intelligente, ma coscienziosa ed amica ${ }^{53}$.

E ancora, pochi giorni dopo:

Matteotti fece la nota delle canagliate per il «primo anno della dominazione fascista ${ }^{54}$. Io vorrei fare l'elenco delle uccisioni negli anni che preparano la marcia su Roma. Il materiale è quasi tutto raccolto: non c'è che metterlo in ordine cronologico, completando per l'Italia meridionale collo spoglio del «Roma» e del «Giornale di Sicilia» e qualche quotidiano sardo. Lavoro non breve e pesante, ma di effetto impressionante ${ }^{55}$.

Il 23 marzo scriveva nuovamente alla moglie di Arfé, dando anche alcune interessanti indicazioni di metodo e chiarendo lo scopo della ricerca:

Vorrei tradurre in italiano un mio libro pubblicato in Inghilterra nel 1928, dedicato alla storia italiana dal 1919 al 1922. Titolo La marcia su Roma ${ }^{56}$. Tradurlo come uscì nel 1928, ma aggiungerci un certo numero di appendici, che confermino e chiariscano le tesi da me affermate allora [...]. In una parola dovrebbe servire a dare un'idea "concreta" di quella che fu la ferocia fascista in quegli anni - ferocia di cui non si ebbe idea allora fuori d'Italia, e non si ha la minima idea oggi in Italia. Il metodo che sono andato escogitando è il seguente. Dare giorno per giorno la lista delle persone ammazzate dai fascisti in tutta Italia: il calendario dei morti: né una parola di più né di meno che le date e i morti. Credo si arriverebbe certo ai 3000 in due anni fino al 28 ottobre 1922 e forse 4000. A questo scopo, fare lo spoglio metodico dei quotidiani nelle diverse parti d'Italia, e ricavarne i nomi dei morti ammazzati. Trascurare i feriti, perché dei feriti non si sa se sono gravi o leggeri: dare i loro nomi so-

${ }_{53}^{3}$ FT, Carte Arfé, Corrispondenza, b. 1, lettera del 10 marzo 1955.

54 Cfr. G. Matteotti, Un anno di dominazione fascista. Numeri, fatti e documenti raccolti a cura della sezione statistica della segreteria del Psu, Tipografia italiana, Roma 1923, poi Forni, Sala Bolognese 1980.

${ }_{55}$ FT, Carte Arfé, Corrispondenza, b. 1, lettera del 22 marzo 1955.

56 In realtà il volume, con il titolo The Fascist Dictatorship, fu pubblicato nel 1927 negli Usa (Henry Holt \& Co., New York) e solo l'anno successivo in Inghilterra (Jonathan Cape, London, trad. it. in Scritti sul fascismo, vol. I, a cura di R. Vivarelli, Feltrinelli, Milano 1966): cfr. ISRT, Archivio Gaetano Salvemini. I manoscritti e materiali di lavoro, inventario a cura di S. Vitali, Ministero per i Beni culturali-Ufficio centrale per i beni archivistici, Roma 1998, pp. 146-47. 
lo quando c'è la notizia che sono morti realmente dopo il fattaccio [...] Insomma, è un lungo e paziente lavoro di riordinamento, che può essere affidato solo a persone di grande coscienza morale. Un solo nome, che non si potesse documentare con esattezza e sicurezza farebbe cadere nel discredito tutto il lavoro [...]. Comincerei col «Giornale di Sicilia», per non sollevare sospetti col «Lavoratore» di Trieste. Ma quando arrivi a questo, devi tener presente che è un giornale comunista, e che per i comunisti la verità è un pregiudizio borghese: quindi, fatte tutte le schede, dovresti prendere il «Piccolo» di Trieste, e controllare se i nomi dei morti si trovano anche lì: se si trovano lì sono sicuri; se non si trovano, metti nella scheda un punto interrogativo: vedremo a tempo debito come regolarci [...]. S'intende che oltre all'elenco dei morti, diciamo così, nostri, dobbiamo fare l'elenco dei fascisti (e non fascisti) uccisi dai nostri; e quelli dei nostri, dei fascisti e non fascisti uccisi dai carabinieri, dalla guardia regia, o dai poliziotti. Insomma un panorama completo delle violenze finite in uccisioni, il quale ci consente di misurare l'estensione del cosiddetto "pericolo bolscevico" di cui i fascisti si vantavano d'aver trionfato, e la sproporzione fra le minacce e la relazione ${ }^{57}$.

Era quindi un'opera «di affetto, di diligenza, di amicizia», con uno scopo importante, «pubblicare una cinquantina di pagine, riempite solamente di numeri, date, sigle, le quali diano un'idea concreta e definitiva di quello che realmente avvenne in quegli anni borgiani» ${ }^{58}$. Ma il lavoro di raccolta e di controllo dei dati proposto da Salvemini dovette rivelarsi fin dall'inizio, agli occhi dello stesso Salvemini, più difficile del previsto e ciò spiega probabilmente perché non fu portato a termine 59 .

\section{Il ritorno alla politica e la Storia dell'Avanti!}

Lavoro storico e impegno politico: il binomio rimane inscindibile, per Arfé, anche in questi anni fiorentini, coerentemente con il «progetto per una politica culturale socialista» inviato a Bosio dopo la morte di Rodolfo Morandi, nel quale riprendeva suggestioni gramsciane e anticipava una discussione che avrà come protagonisti, nell'area socialista, Fortini e Guiducci:

Il Psi ha bisogno di elaborare una propria linea di politica culturale che si proponga i seguenti fini: a) avviare in seno alla sinistra italiana un processo di ampia e libera discussione, che abbia carattere non eccezionale, ma permanente b) promuovere la

57 FT, Carte Arfé, Corrispondenza, b. 1, lettera ad Anna Pagliuca del 23 marzo 1955. Per una recente discussione del tema del fascismo come "controrivoluzione preventiva", con riferimenti anche alle posizioni di Salvemini, cfr. P.G. Zunino, Salvatorelli, la prima storiografia sul fascismo e il problema delle origini della dittatura, in A. d'Orsi (a cura di), Luigi Salvatorelli (1886-1974), Aragno, Torino 2008, pp. 405-50.

58 FT, Carte Arfé, Corrispondenza, b. 1, lettera ad Arfé del 2 aprile 1955.

59 Nelle Carte Salvemini restano solo alcuni appunti sullo squadrismo fascista (cfr. Archivio Gaetano Salvemini cit., p. 143) e non ho rintracciato le schede citate, che pure furono in parte utilizzate per le Lezioni di Harvard. 
formazione di organismi culturali ad alto livello che siano in grado di farsi strumenti di elaborazione e di realizzazione della politica culturale, in stretta collaborazione con la Direzione del partito c) promuovere entro i limiti del possibile una produzione culturale a carattere scientifico. Il partito nel suo complesso non potrà più fare alcun passo avanti se non si eleverà tutto il suo livello culturale. Il compito dei dirigenti politici sarebbe in queste attività ridotto a quello di partecipanti, di "pares inter pares", senza portarvi cioè né diffidenze, né pretese di incompetenze (sic). Nulla di più falso e di più dannoso del dualismo tra "intellettuali" e "politici". Nel partito non esistono "intellettuali" e "politici", ma buoni e cattivi compagni, dotati di varia attitudine e di varie capacità, ma tutti con gli stessi diritti e gli stessi doveri. Chi lavora in organismi culturali e perciò al tempo stesso intellettuale e politico ${ }^{60}$.

Si avvicinava il '56, il XX Congresso e gli avvenimenti ungheresi, con le inevitabili ripercussioni nel mondo della sinistra. Arfé vi fu direttamente coinvolto:

La svolta autonomistica nel 1956 era già nell'aria, ma essa presupponeva anche una rivalutazione critica di tutta la tradizione socialista rinnegata o ignorata, e a Nenni parve che la storia dell' «Avanti!» - il suo «Avanti!» - del quale cadeva in quell'anno il sessantesimo anniversario, potesse fornire l'occasione migliore a una operazione che rispondeva a quel che Bosio definiva, con espressione hegeliana, un bisogno dei tempi ${ }^{61}$. L'incarico di trovare chi scrivesse questa storia fu da Nenni affidato a Raniero Panzieri, allora responsabile della politica culturale del partito ${ }^{62}$

${ }^{60}$ Cfr. la bozza di progetto in IMSC, Carte Bosio, b. 127.

${ }^{61}$ Era un bisogno che Bosio sentiva ormai da tempo. Dopo l'esperienza di «Movimento operaio» aveva infatti progettato, con le Edizioni Avanti!, una Storia del movimento operaio «monografica, popolare, illustrata», di cui inviò il progetto il 13 febbraio 1954 ad Arfé, cui propose di scrivere il volume XV (Il movimento operaio e la lotta politica durante il periodo giolittiano) e il volume XVIII (La crisi del primo dopoguerra e il movimento operaio 19181922). Tra gli altri possibili collaboratori della serie Bosio indicava, oltre alla moglie di Arfè, Anna Pagliuca, anche Luigi Cortesi, Saverio Francesco Romano, Franco Catalano, Gaetano Cingari, Giovanni Pirelli, Maurizio Vitale, Stefano Merli, Renato Carli Ballola, Domenico Zucàro (cfr. la lettera di Bosio, con allegato il piano dell'opera, in FT, Carte Arfé, Corrispondenza, b. 1). Arfé aveva anche in previsione di pubblicare, nella collana "Saggi e documentazioni" delle Edizioni Avanti! una Storia della Federazione Giovanile Socialista, come testimonia la lettera a Bosio dell' 8 gennaio 1954: «Sto rifacendo ancora una volta la prima parte della storia della Fgs. Mi sono accorto che non era sufficientemente legata alla storia del partito. Vorrei, nei limiti del possibile e delle mie capacità, fare un lavoro pulito» (IMSC, Carte Bosio, b. 85). Arfé aveva chiesto consiglio anche a Salvemini, che il 31 gennaio 1956 gli aveva così risposto: «Ho paura che troverai assai poco di interessante nel movimento giovanile socialista italiano prima del fascismo (e durante e dopo). C'era poco pensiero nei vecchi: immaginarsi nei giovani! Naturalmente, erano tutti "estremisti": ma essere estremisti ha mai significato avere in testa qualcosa?» (FT, Carte Arfé, Corrispondenza, b. 1). Il volume uscirà nel 1973 per le Edizioni del Gallo col titolo Il movimento giovanile socialista. Appunti sul primo periodo 1903-1912.

62 Così lo descrive Arfé, chiarendo anche il senso dell'operazione culturale (e politica) compiuta con la Storia dell'Avanti!: «Un giovane intellettuale che aveva abbandonato per la milizia di partito la carriera universitaria, che aveva già aperto la prima breccia nel conformismo imperante con la riscoperta e la rivalutazione dei primi scritti di Morandi, il Morandi anti- 
[...]. A Bosio si rivolse Panzieri, a Giovanni Pirelli e a me, che delle stesse vicende eravamo stati partecipi, a Domenico Zucàro, a qualche altro compagno, e ci convocò tutti presso la Direzione del partito. Eravamo agli inizi della primavera e la storia del giornale, per categorica richiesta di Nenni, doveva essere pronta per la fine d'agosto, onde poter essere diffusa nel corso delle feste dell' «Avanti!» di settembre, che preludevano alla campagna congressuale. Lo stesso Panzieri, abitualmente portato ad affrontare le cose con signorile flemma, era stato colto da un impeto di attivismo intellettuale e pratico, e premeva perché quanto prima una presenza socialista, con propria autonomia ideale, si manifestasse in campo storiografico ${ }^{63}$.

$\mathrm{Fu}$, come si può immaginare, un lavoro faticosissimo 64 , per i tempi ristretti, l'enorme mole di carta stampata da esaminare, lo stato assai carente degli studi sul socialismo italiano. La storia del giornale fino alla sua soppressione nel 1926 apparve comunque ai primi di settembre (il secondo volume, fino al 1940, fu pubblicato l'anno dopo) e Arfé poté annunciarne l'uscita a Salvemini (con il quale aveva discusso, nell'impostazione del volume, su un tema, quello dell'interventismo cui Salvemini era, evidentemente, particolarmente sensibile) ${ }^{65}$ :

Sono stato lungo tempo senza farmi vivo perché sono stato impegnatissimo con una «Storia dell'Avanti!» che mi è toccato scrivere in pochissimi mesi e che mi ha costretto a scorrere tutta la collezione del giornale, a partire dal 1896. Credo che a quest'ora le sia già arrivata una copia del volumetto. La mia preoccupazione è stata quella di fare un lavoro onesto che sia utile ai non intellettuali che lo leggeranno ${ }^{66}$.

Sono preoccupazioni che emergono anche nella lettera a Nenni del 30 agosto 1956:

statalista e antiburocratico del Centro Interno, il Morandi dei Consigli di gestione. La scherzosa parola d'ordine lanciata in privato da Panzieri era quella di "organizzare il disordine", di rompere coraggiosamente i vecchi schemi, di mettere in contatto, di stabilire canali permanenti di comunicazione tra gruppi formatisi su esperienze diverse, senza preoccuparsi di precostituire ortodossie e di prefissare traguardi. Devo a lui e a Bosio - la richiesta politica era partita da Nenni - l'avere scritto nel '56 una Storia dell'Avanti!, la quale voleva essere nelle nostre comuni intenzioni prima ancora che rivalutazione critica della assai vilipesa tradizione socialista, rottura di una ideologia storiografica di marca comunista riassumibile nella formula che soltanto con la scissione di Livorno il movimento operaio italiano passava dalla preistoria alla storia, che tutto il periodo antecedente non era che faticosa preparazione, fitta di errori e di colpe, al gran giorno la cui alba era sorta nel gennaio del 1921» (Intellettuali e società di massa: i socialisti italiani dal 1945 ad oggi, Ecig, Genova 1984, p. 36).

63 G. Arfé, Premessa alla seconda edizione della Storia dell' "Avanti!", Mondo OperaioAvanti!, Roma 1977.

64 Scriveva nel maggio 1956 a Bosio: «Caro Gianni, ti prego di farmi avere indicazioni e notizie relative alla fondazione dell'"Avanti!". Sto procedendo alla prima stesura della prima parte e vorrei aver sott'occhio tutti gli elementi. Lavoro da negro!» (IMSC, Carte Bosio, b. 85).

65 «Egli non mi oppose le sue verità, ma fu indotto, mi disse, a riflettere ancora, fino al punto da commissionare una ricerca sul dibattito pro e contro l'intervento sui maggiori giornali italiani. Non cambiò opinione, ma riconobbe che Turati aveva qualche ragione quando aveva definito lui e i suoi amici interventisti "i raggirati della storia"»(G. Arfé, La legge del non mollare cit.).

${ }^{66}$ Lettera del 17 ottobre 1956, in ISRT, Carte Salvemini, b. 92. 
La difficoltà maggiore che ho incontrata è stata quella di dover rivedere tutta una certa interpretazione della storia del socialismo italiano dal 1921 in poi, mantenendo serenità di giudizio ed evitando toni apertamente polemici. Tra gli ostacoli che mi sono trovati di fronte c'è stato quello di carattere metodologico, consistente nel fatto che, proprio per questo periodo, la storia dell' «Avanti!» è strettamente fusa con quella del partito, il che mi ha reso difficile approfondire il discorso senza uscire dai limiti dell'argomento. A questo si aggiunge che la revisione del problema storiografico sarebbe dovuta andare di pari passo con una revisione dei problemi ideologici relativi, cosa che mi avrebbe portato su di un terreno che ho preferito non toccare in un caso come questo, in cui il mio lavoro si svolgeva nell' ambito di un'iniziativa di partito. Se però la mia impostazione della nostra storia non ha carattere compiuto, credo che vi siano molti spunti sui quali si potrà sviluppare una utile discussione ${ }^{67}$.

Il notevole successo del libro (e le sue ripercussioni in campo storico e politico ${ }^{68}$ ) spinsero Arfé a tornare alla politica (che peraltro, come abbiamo visto, non aveva mai abbandonato), ritorno che annunciò in quella che sarà l'ultima lettera a Salvemini:

Devo confessarle che mi sono rimesso a «far politica». Mi è parso che la situazione permetta di fare qualche cosa e ho cominciato a darmi da fare. È un morbo, Lei lo sa bene, dal quale non si guarisce [...]. Ma d'altra parte quando si è convinti di avere qualche cosa da dire, di avere la possibilità, anche remota, di cambiare in meglio qualche cosa, si finisce col non resistere alla spinta. Nel «Ponte» di dicembre troverà un mio articolo politico ${ }^{69}$. Son le cose che sto cercando di discutere nel partito socialista, ma quel che è più difficile è che poi dalle discussioni si passi alle realizzazioni e che si organizzi una attività seria di studio sui concreti problemi ${ }^{70}$.

La risposta di Salvemini fu, come spesso gli capitava con gli amici, tra l'ironico e l'affettuoso: «Quanto alle attività politiche tue, che cosa vuoi che

${ }^{67}$ ACS, Carte Nenni, b. 17.

68 «Tra le molte presentazioni ricordo quella di Roma, fatta da Raniero Panzieri e da un brillantissimo e polemico Fernando Santi, il quale partì dai suoi rapporti con Turati per arrivare all'ostracismo di cui era stato vittima il socialismo riformista con l'acquiescenza dello stesso Nenni. A Milano la presentazione fu organizzata da Rossana Rossanda presso la Casa della Cultura, dove il tema principale fu quello della nuova fase del rapporto tra socialisti e comunisti. A Napoli fu Francesco Compagna ad organizzare la manifestazione e Vittorio De Caprariis, proprio in quell'occasione, salutò il sorgere di una storiografia socialista autonomista. Tra le recensioni ricordò quella dell' «Unità», scritta da Lucio Lombardo Radice, che parlava della «scoperta del tesoro» della tradizione socialista da inserire nella cultura storica e politica dei due partiti della classe operaia. A Firenze venne a trovarmi anche Bettino Craxi, che ancora non conoscevo. Era un giovane spilungone avvolto in un cappotto stinto e, nell'esprimermi il suo compiacimento, mi incitò a scendere in campo nel congresso ormai imminente, promettendomi tutti i voti di cui poteva disporre per l'elezione al Comitato centrale» (G. Arfé-A. Ricciardi, Dialogo sul socialismo cit., p. 98).

69 G. Arfé, Il socialismo italiano tra il passato e l'avvenire, «Il Ponte», dicembre 1956, pp. 2041-49.

${ }^{70}$ Lettera del 31 dicembre 1956, in ISRT, Carte Salvemini, b. 92. 
ti dica? Fa quello che credi sia il tuo dovere, ma fallo con buon senso e senza esagerare in... entusiasmo» 71 .

Salvemini morì a Sorrento il 6 settembre 1957, laicamente e circondato dall'affetto degli amici, come ad Arfé, in una sorta di prefigurazione, toccò spesso di ricordare:

Ho varcato la soglia della vecchiaia e ho scoperto che la si può vivere, anche da laici, in stato di grazia, quello che si raggiunge quando si può guardare al passato, senza superbia, che è peccato, ma con l'intimo convincimento di aver conservato il rispetto di se stessi e la stima e l'amicizia delle persone che incontrammo lungo la nostra via, di poter dialogare ancora idealmente con loro, di poter immaginare i loro consigli, di poter sentire ancora il calore del loro affetto. C'è una eutanasia che è difesa della propria dignità umana quando si può essere non più in grado di salvarsela da soli e può diventare necessario ricorrere a mani esperte e pietose e ce ne è una che è tutta e solo nelle nostre mani. Il caso ha voluto che a insegnarmelo - l'ho capito col passar degli anni - sia stato il padre spirituale degli "azionisti”, Salvemini, che morì dicendosi felice di avere intorno a sé tanti amici i quali non avevano le sembianze di compunti e sussiegosi colleghi, ma erano i suoi compagni di avventure, di fede e di passione, i vivi e i morti. Spero - la speranza è virtù - che tocchi anche a me lo stesso destino ${ }^{72}$.

\section{Riflessioni salveminiane}

Arfé continuerà a riflettere sugli scritti di Salvemini anche dopo la morte di quest'ultimo, a partire dalla ricordata Prefazione a Movimento socialista e questione meridionale. Ciò che lo interessava era la natura del socialismo salveminiano ( «rifondazione politica prima che sociale, scettico di fronte alla ineluttabilità delle palingenesi totale, ma non alieno dal ricorso a tutte le armi per conquistare graduali riforme» ${ }^{73}$ ) e le sue differenze con quello turatiano.

Si può però forse affermare che, per certi versi, la sua attenzione fosse attratta ancora di più dalle riflessioni salveminiane sui partiti politici, la loro organizzazione e il loro ruolo nella società contemporanea, dovendone riconoscere la fondamentale fondatezza dell'intuizione sulla natura sostanzialmente "conservatrice" dei partiti, nella loro tendenza «a cristallizzarsi in organizzazioni di interessi economici e politici» che, solo se costantemente pungolati da gruppi sciolti da ogni vincolo disciplinare, in grado di mettere in circolazione idee e programmi, possono riuscire ad elaborare delle soluzioni per determinati problemi e promuoverne la realizzazione ${ }^{74}$.

Il tentativo di costruire strumenti di questo genere sarà portato avanti più volte da Arfé, nel corso della sua carriera politica, soprattutto all'epoca del

\footnotetext{
${ }^{71}$ FT, Carte Arfé, Corrispondenza, b. 1.

72 G. Arfé, Lettera aperta a Indro Montanelli cit.

${ }^{73}$ Id., Prefazione cit., p. XII.

74 Ibidem.
} 
primo centro-sinistra, in collaborazione con una notevole figura di intellettuale-tecnico, Roberto Guiducci:

Ho avuto un lungo colloquio con Guiducci, che si è impegnato tra l'altro a costituire a Milano un gruppo di collaboratori fissi di «Mondo Operaio». Questo legame però avrà un senso, e continuerà, soltanto se diventerà in qualche misura operante sul terreno politico. Sono refrattario per formazione alle infatuazioni tecnicistiche, e con Guiducci stesso, in più di una occasione, ho amichevolmente polemizzato. Ma il suo discorso attuale nella sostanza è tutt'altro che astratto, e mi ha confermato la sua convinzione, tratta già da altri sintomi e da altre esperienze, che siamo arrivati a un punto critico nei rapporti con le correnti e con le forze che ci hanno fin qui sostenuti. Le circostanze in questo momento sono, a mio avviso, favorevoli ad una ripresa di contatto, ma se questa mancasse, arriveremmo rapidamente al punto di rottura ${ }^{75}$.

$\mathrm{Fu}$, come è noto, un tentativo fallito e «al punto di rottura» si arrivò molto rapidamente ${ }^{76}$. Tra i molti motivi della frattura tra partiti e società (o della rispettiva omologazione), non ultimo sarà il valore degli uomini che, dopo la generazione dell'antifascismo e della Resistenza, si troveranno a ricoprire i più alti incarichi di governo e di partito. Ancora una volta tornava un insegnamento salveminiano:

Agli uomini perciò, non alle forze oscure dell'economia, egli affidava il suo insegnamento e le sue speranze. La proposta con cui egli chiudeva la prefazione ai suoi scritti di mandare nelle regioni del Sud i migliori docenti italiani, poteva, egli stesso lo riconobbe, sembrare anche ingenua; ma dietro di essa era la sua fede che dove si seminano idee, dove si insegna con l'esempio l'onestà senza aggettivi, il raccolto non può mancare, perché sempre e solo gli uomini sono i protagonisti della storia ${ }^{77}$.

75 G. Arfé a P. Nenni, lettera del 12 novembre 1964, in ACS, Carte Nenni, b. 17.

76 Scriverà Nenni nei suoi Diari il 26 settembre 1971, alla fine di un colloquio con Arfé: «Il quadro che Arfé fa del partito è agghiacciante. La politica non fu mai cosa da prima comunione ma neppure raggiunse mai tali livelli, quelli propri dell'attuale società dell'edonismo e del consumismo. Un consumo anche la politica!» (P. Nenni, Diari, vol. III, I conti con la storia 1967-1971, SugarCo, Milano 1983, p. 635).

77 G. Arfé, Prefazione cit., p. XV. 
